\title{
Glottopol
}

Revue de sociolinguistique en ligne

$33 \mid 2020$

Varia - Liberté, égalité, diversité !

\section{Caractérisation de la variation liée à la situation de communication : apport de l'acoustique à la phonostylistique}

Mélanie Lancien

\author{
(2) OpenEdition \\ Journals \\ Édition électronique \\ URL : https://journals.openedition.org/glottopol/609 \\ DOI : $10.4000 /$ glottopol.609 \\ ISSN : 1769-7425 \\ Éditeur
}

Presses universitaires de Rouen et du Havre

Référence électronique

Mélanie Lancien, « Caractérisation de la variation liée à la situation de communication : apport de l'acoustique à la phonostylistique », Glottopol [En ligne], 33 | 2020, mis en ligne le 01 janvier 2020, consulté le 02 octobre 2021. URL : http://journals.openedition.org/glottopol/609 ; DOI : https:// doi.org/10.4000/glottopol.609 


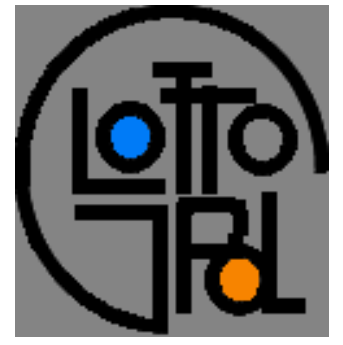

\section{GLOTTOPOL}

Revue de sociolinguistique en ligne n`33 - février 2020

Varia : Liberté, égalité, diversité !

\section{SOMMAIRE}

Hommages à Jean-Baptiste Marcellesi : À ringrazià vi, par Niculau Sorba ; "Ciao Ziu ! » par Philippe Blanchet.

Clara Mortamet : Présentation du numéro : Liberté, égalité, diversité !

Manon Him-Aquilli : "Prendre la parole sans prendre le pouvoir ». La mise en registre de l'horizontalité dans les assemblées générales anarchistes/autonomes.

Siham Hocini : La formulation du désaccord dans les débats télévisés algériens : quels "effetsde-politesse "?

Pauline Rannou: Trajectoires de la surdité en France. Parents entendants d'enfants sourds: questionner la fragmentation de l'identité sourde.

Catherine Combaz-Champlaine: Les rectifications orthographiques de 1990 comme révélateurs du rapport des enseignants à l'orthographe.

Marie-Madeleine Bertucci : Éléments d'épistémologie pour l'étude des espaces ségrégués des banlieues urbaines sensibles de la région parisienne.

Élise Gandon: Usages du numérique et illectronisme: nouvelles tâches pour le lecteurscripteur, nouveaux enjeux d'apprentissage.

Marc Arabyan : Quelle langue pour la recherche neurolinguistique française?

José R. de Arellano : Le rapprochement entre l'espagnol et le portugais pendant la décennie dorée sud-américaine : une problématique de l'horizontalité supercentrale.

Adam Wilson: Normes interactionnelles globalisées et communautés de pratique discontinues : les dynamiques sociolinguistiques du tourisme international.

Mélanie Lancien : Caractérisation de la variation liée à la situation de communication : apport de l'acoustique à la phonostylistique.

Assémou Maurice Ludovic Assémou : La prononciation du français parlé en Côte d'Ivoire.

http://glottopol.univ-rouen.fr 


\title{
CARACTÉRISATION DE LA VARIATION LIÉE À LA SITUATION DE COMMUNICATION : APPORT DE L'ACOUSTIQUE À LA PHONOSTYLISTIQUE
}

\author{
Mélanie Lancien \\ Section des Sciences du Langage et de l'Information, Université de \\ Lausanne (Suisse)
}

\section{Introduction}

La plupart des études sur la variation phonique menées à partir des années '70, notamment celles de Labov (1972) sur les variables sociales liées à la prononciation des /R/ à New-York, ou de Trudgill \& Trudgill (1974) sur différents phones (dont les /R/) en Norvège, ont été faites sur une base perceptuelle : la qualité des sons était déterminée par l'oreille des chercheurs. À l'aube des années 2000, les techniques de la phonétique acoustique se sont mises au service de la sociolinguistique pour apporter des mesures physiques, objectives, qui complétaient les intuitions auditives des chercheurs. C'est notamment ce qui a permis l'émergence des études sur la qualité des voyelles diphtonguées de Labov (1990). Cependant les mesures spectrales étaient délicates à obtenir et à analyser avant l'apparition des logiciels de traitement de la parole (Praat, Winpitch, Wavesurfer, par exemple). Au-delà des difficultés techniques, des difficultés méthodologiques s'ajoutent. Entre autres: une étude expérimentale visant à déterminer l'impact de variables sociales sur la prononciation doit s'assurer autant que possible de préserver la même scène (le lieu, les observateurs externes, le moment, le type d'activité de communication, et le but de l'activité de communication) pour tous les locuteurs enregistrés. Il est également nécessaire de garder le même matériel phonique (mêmes phrases répétées par tous les locuteurs), et de fournir des enregistrements sonores de qualité suffisante pour les analyses phonétiques afin de pouvoir tirer le maximum d'informations d'une analyse acoustique, et assurer sa fiabilité.

Le développement de grands corpus oraux a permis de donner une assise plus solide aux observations acoustiques faites sur la variation sonore, par exemple en apportant des données plus représentatives (notamment par le nombre d'observations), et en systématisant les analyses de la qualité acoustique des phones et en les croisant avec des variables sociales grâce à des modèles statistiques qui permettent d'évaluer le poids de chaque variable sociale sur la variation des sons. C'est par exemple le cas dans une étude de Adda-Decker \& Lamel (1999), qui ont mis en évidence les interactions entre la réduction acoustique des mots, la fréquence de ces mots, et le style de parole dans lequel ils sont prononcés (lu vs. spontané). Ou encore d'une 
étude de Devilla \& Trimaille (2010 ; voir aussi Vernet \& Trimaille, 2007 ; Trimaille et al., 2012), qui a démontré que l'exploitation de la variation phonique par les locuteurs peut avoir des fins identificatrices au sein d'une communauté de pratique. Cette série d'études visant à observer les phénomènes d'affrication dans l'accent de banlieue a montré une tendance à prononcer les consonnes occlusives dentales [t] et [d] comme des affriquées ([ts] et [dz]) chez les jeunes de banlieue (notamment la banlieue grenobloise et parisienne) ${ }^{1}$, cette variante de prononciation prenant alors une fonction identificatrice pour les locuteurs qui la réalisent aussi bien que pour les membres d'autres communautés de pratique qui y sont exposés.

De plus, le big data, que nous définissons ici principalement via la caractéristique de la masse (on entre dans le big data à partir du moment où la masse de données est telle qu'une automatisation de son traitement est absolument nécessaire ${ }^{2}$ ), permet aujourd'hui à la phonétique d'assouplir le degré de contrôle des productions. Par exemple, si l'étude de la qualité vocalique nécessite le contrôle du contexte consonantique des voyelles lorsque l'on observe un jeu de données de petite taille, ce contrôle peut être réduit lors d'une étude de grande ampleur, une simple prise en compte de l'impact du contexte consonantique devenant alors suffisante (Gahl, 2015).

L'accessibilité des grands corpus (la création et mise à disposition de corpus de parole médiatique annotés automatiquement, par exemple), l'assouplissement du contrôle des données acoustiques, et le développement d'outils permettant de croiser les informations acoustiques et les informations sociolinguistiques (nous pensons par exemple aux modèles statistiques tels que les modèles linéaires mixtes), conduisent à des collaborations de plus en plus étroites entre phonétique et sociolinguistique. On observe d'ailleurs depuis quelques années l'émergence d'une discipline appelée "sociophonétique", qui porte un intérêt tout particulier à l'observation des différences diamésiques, diaphasiques, diastratiques, et diatopiques dans la production et la perception de la parole. Cette discipline est perçue de différentes façons par la communauté scientifique, tantôt comme une simple extension de la phonétique à l'étude de variables sociales (Baranowski, 2013), ou comme un emprunt d'outils permettant à la sociolinguistique de prolonger les études sur la variation commencée par Labov (Hay \& Drager, 2007 ; voir Candea \& Trimaille, 2015 pour une revue plus exhaustive). Ici nous la considérons comme une collaboration, une rencontre entre deux domaines, bien que nos méthodes soient davantage héritées de la phonétique que de la sociolinguistique. Ces collaborations permettent de reproduire les études perceptives menées au siècle dernier, mais également de les pousser plus loin, d'observer des variables plus fines, qui ne sont pas toujours perçues par les chercheurs (comme par exemple la réduction spectrale des phones). La phonétique permet notamment aujourd'hui de placer les voyelles diphtongues sur des continuums indiquant à quel point ces voyelles diphtonguent et quel est leur point de départ et d'arrivée, ce que Labov (1990) avait entamé dans ses études sur le lien entre la variation diastratique et la diphtongaison des voyelles en anglais américain.

C'est de cette façon que les premières études de phonostylistique sur les différences entre la parole lue et la parole spontanée (Harmegnies \& Poch-olivé, 1992 ; Poch-Olivé \& Harmegnies, 1992 ; Adda-Decker \& Lamel, 1999 ; Rouas et al., 2010) ont mis en évidence un phénomène d'hypoarticulation (Lindblom, 1990) dans la parole spontanée : les voyelles sont plus courtes et plus centralisées (leur qualité est réduite et tend vers celle d'un schwa) en parole spontanée que dans des conditions de lecture. Lors d'interactions spontanées, l'alternance entre styles est guidée par différents aspects de la situation de communication (Labov, 1972 et Eskenazi, 1993

\footnotetext{
${ }^{1}$ Des études plus récentes montrent cependant que cette affrication se généralise en France, et se retrouve désormais dans les médias.

${ }^{2}$ cf. la présentation de Bruno Bachimont à la $2^{\mathrm{e}}$ journée d'étude « Méthode, intégrité scientifique \& données » (16 février 2018) disponible à l'adresse suivante : https://commondata.fr/2018/04/03/bruno-bachimont-les-big-dataou-la-necessite-de-passer-dune-epistemologie-de-la-mesure-a-celle-de-la-donnee/
} 
mentionnaient principalement le sujet de la discussion et le statut des interlocuteurs). Parmi ces aspects, on compte notamment les variables liées aux participants de l'interaction, telles que l'identité de l'interlocuteur et la nature des relations entre individus. Ces caractéristiques individuelles des interactants ont déjà été décrites comme des facteurs de variation phonétique. Scarborough et al. (2007, 2013), ainsi que Koppen (2017), pour les études les plus récentes, ont montré que le degré de formalité de l'interaction, la présence physique de l'auditeur, sa langue maternelle ou la présence d'une déficience auditive chez l'un des participants influencent effectivement la qualité acoustique des voyelles, le degré d'hypoarticulation (Lindblom, 1990), ainsi que le débit de parole. En d'autres termes, les locuteurs d'une langue donnée adaptent leur débit et leur effort articulatoire en fonction de l'identité de leur interlocuteur.

Nous proposons ici de mettre les méthodes de la phonétique de grand corpus au service de la description des styles de parole, de façon à mettre en évidence des paramètres acoustiques sociolinguistiquement pertinents pour l'analyse des styles de parole.

Dans la veine de Labov (1990) nous proposons d'examiner ici les productions de 10 locuteurs dans quatre styles de parole chacun : la lecture de listes de mots, la lecture de texte, la discussion avec une expérimentatrice, et la discussion entre conjoints. Dans cette étude nous adaptons la typologie des styles de parole établie par Labov (1966). Au lieu de considérer cinq styles : la parole vernaculaire, la parole surveillée, la lecture, la lecture de listes de mots, la lecture de paires minimales; nous proposons une division en deux macro-styles ${ }^{3}$ : la parole spontanée et la lecture, qui se divisent en sous-styles en fonction des différentes composantes de la condition de production (dans notre cas, l'identité de l'interlocuteur pour la parole spontanée, et le support fourni pour la lecture). Nous examinerons les quatre styles énumérés ci-dessus sous le microscope de l'hypoarticulation des voyelles (Lindblom, 1990), en prenant en compte un ensemble de mesures temporelles et spectrales caractérisant les voyelles produites dans chaque style. Ces mesures permettront d'évaluer l'atteinte des cibles articulatoires, soit le fait que lors de l'articulation le «point d'équilibre $»^{4}$ caractéristique du segment lorsqu'il est «neutre articulatoirement $»^{5}$ (dans notre cas en situation de lecture de mots) soit atteint, révélatrice de l'attention portée à l'articulation lors de la production des sons, ainsi que la vitesse d'élocution, dans une moindre mesure. Nous proposons également de dépasser la simple distinction lecture vs. parole spontanée par l'examen sociophonétique du lien entre la distance sociale entre deux locuteurs et le degré d'hypoarticulation (Lindblom, 1990) dans des interactions spontanées entre inconnus et entre conjoints.

S'il a déjà été démontré que la parole formelle montre moins de réduction que la parole informelle du point de vue du débit articulatoire (Koppen et al., 2017), nous nous attacherons à montrer que la distance sociale influence également les caractéristiques acoustiques des voyelles. Dans un premier temps, nous considérons l'hypo-hyperarticulation comme un moyen de transmettre la distance sociale dans les relations humaines. Plus précisément nous testons l'hypothèse selon laquelle plus la distance sociale avec l'interlocuteur est grande, plus le locuteur aura tendance à l'indiquer par une hyperarticulation ; a contrario, plus la distance est faible plus le locuteur tendra vers l'hypoarticulation. Nous démontrerons ensuite que, bien que la parole adressée à un proche soit sous certains aspects plus hypoarticulée, il est nécessaire de sortir la réflexion du cadre linéaire de l'hyper-hypoarticulation pour rendre compte de la complexité du phénomène.

Cette étude, bien que menée uniquement sur les productions de 10 locuteurs suisses romands, a permis de récolter plus de 25000 réalisations de phones. Par conséquent, nous utilisons les techniques de la phonétique de grand corpus, et menons des analyses quantitatives

\footnotetext{
${ }^{3}$ Aussi appelé « phonogenre » dans sa théorisation par Mathon (2014).

${ }^{4}$ Terme repris de Fougeron (2005).

${ }^{5}$ Terme repris de Lindblom et Sunberg (1971).
} 
de la qualité et de la longueur des voyelles en fonction des styles de parole, de la distance sociale entre interactants, et de trois marqueurs sociaux plus classiques : l'âge, le genre, et l'origine régionale. Le français suisse romand a très peu été étudié du point de vue phonostylistique, c'est pourquoi nous reproduisons dans un premier temps les résultats classiques concernant la variation entre la lecture et la parole conversationnelle : la parole conversationnelle montre une plus forte réduction vocalique, un plus grand degré d'hypoarticulation que la lecture de mots, à la fois sur le plan temporel et sur le plan spectral. En revanche, notre étude montre un plus grand degré de complexité qu'un simple positionnement des styles de parole sur l'axe de l'hyper-hypoarticulation : bien que les situations de communication dans lesquelles sont placés nos locuteurs et nos locutrices restent très stéréotypées, nos résultats montrent que lorsque l'on dépasse la simple opposition entre les macro-styles lecture/parole spontanée, une approche linéaire de l'hyper-hypoarticulation n'est plus suffisante pour pouvoir décrire le degré de réduction présenté par les styles de parole. Il nous faut alors étudier la combinaison des différentes caractéristiques phonétiques de la parole au sein de chaque style pour pouvoir rendre compte de la variation entre ces styles, et des caractéristiques qui leur sont propres. En somme, cet article montre le besoin de proposer de nouveaux modèles non-linéaires de la variation stylistique prenant en compte (entre autres) des aspects interactionnels et plus de paramètres acoustiques que la durée vocalique et la dispersion des phones au sein du système ${ }^{6}$.

\section{Méthodologie}

\section{Corpus de travail}

Ce travail s'appuie sur des données récoltées au sein du projet PFC (Phonologie du Français contemporain; Durand et al., 2002). Le Projet PFC est un programme de recherche pluridisciplinaire visant à décrire certains phénomènes linguistiques présents dans le français parlé. Depuis les années 2000, le projet collecte des données dans toute la francophonie : France, Belgique, Suisse, Canada, Afrique du nord (entre autres). Actuellement, environ 1000 locuteurs et locutrices du français ont été enregistrés au sein du projet PFC. La majeure partie de ces données sont encore en cours de traitement.

Le protocole d'enregistrement mis en place comprend l'enregistrement de tâches de lecture et de tâches de conversation. Chaque locuteur et locutrice lit à haute voix une à trois listes de mots, ainsi qu'un texte, une conversation dite " guidée » avec un enquêteur, et une discussion dite « libre » avec un membre de la même communauté linguistique (généralement un ami ou un membre de la famille) sont également réalisées.

Les locuteurs et locutrices sont choisis sur la base de critères précis. Une zone géographique restreinte (en générale une ville) est ciblée comme point d'enquête, chaque locuteur et locutrice doit être originaire de cette zone et y avoir passé la majorité de sa vie. Pour chaque point d'enquête, environ six femmes et six hommes sont interrogés. Sur cette douzaine de locuteurs et locutrices, lorsque cela était possible deux de chaque genre appartenaient à la tranche d'âge 20-39 ans, deux appartenaient à la tranche d'âge 39-59 ans, et enfin deux avaient 60 ans et plus.

Dans la présente recherche, nous nous sommes concentrée sur les données issues du souscorpus suisse du projet PFC (Andreassen et al., 2010 ; Racine et Andreassen, 2012, ; Andreassen et Racine, 2016) ${ }^{7}$. Notre jeu de données comporte dix locutrices et locuteurs natifs de Suisse romande (cinq femmes et cinq hommes). Le choix de ces locuteurs et locutrices s'est principalement fait en fonction de la relation qu'ils entretenaient avec à la fois l'enquêtrice

\footnotetext{
${ }^{6}$ Mesure de distance spectrale permettant de localiser les phones dans un espace acoustique F1/F2, généralement utilisée pour mesurer la réduction vocalique

${ }^{7}$ https://public.projet-pfc.net/enquetes.php?id=144
} 
impliquée dans la conversation guidée, et l'interlocuteur impliqué dans la conversation libre. Pour augmenter le contraste, nous avons veillé à ce que toutes les conversations libres aient lieu entre mari et femme et à ce que l'enquêté(e) ne connaisse pas l'enquêtrice ${ }^{8}$ en conversation guidée. Dans le choix de nos données, nous n'avons cependant pas pu prendre en compte la tranche d'âge à laquelle appartiennent les locuteurs et locutrices. Le détail des caractéristiques des locuteurs et locutrices sélectionné(e)s est présenté dans le tableau 1. Notre corpus de travail est constitué de 3,5 heures d'enregistrements de discussions, 3956 mots lus, et 10 textes lus $(=28,8$ min de lecture $)$.

\begin{tabular}{|l|l|l|l|l|l|}
\hline Code Locuteur & Genre & Point d'enquête & Âge & $\begin{array}{c}\text { Relation } \\
\text { avec l'interlocuteur } \\
\text { de la discussion guidée }\end{array}$ & $\begin{array}{c}\text { Relation } \\
\text { avec l'interlocuteur } \\
\text { de la discussion libre }\end{array}$ \\
\hline RV1 & M & Martigny & 79 & Inconnu & Mariage \\
\hline LV1 & F & Martigny & 78 & Inconnu & Mariage \\
\hline PG1 & M & Martigny & 59 & Inconnu & Mariage \\
\hline PG2 & F & Martigny & 58 & Inconnu & Mariage \\
\hline SD1 & F & Martigny & 32 & Inconnu & Mariage \\
\hline FD1 & M & Martigny & 42 & Inconnu & Mariage \\
\hline PM1 & M & Neuchâtel & 42 & Inconnu & Mariage \\
\hline CM1 & F & Neuchâtel & 39 & Inconnu & Mariage \\
\hline PY1 & M & Neuchâtel & 44 & Faible connaissance & Mariage \\
\hline CY1 & F & Neuchâtel & 43 & Inconnu & Mariage \\
\hline
\end{tabular}

Tableau 1 : Récapitulatif des caractéristiques des locuteurs et locutrices inclus dans notre corpus de travail

La variété de français parlée en Suisse romande comporte un inventaire vocalique relativement semblable à celui du français de référence (Lyche, 2010), avec 14 à 16 voyelles phonologiques. À la différence que le français suisse romand conserve la distinction entre les voyelles phonologiquement longues /i:, y:, e:, a:, $\varepsilon$ :, u:/ et les voyelles phonologiquement brèves $(/ \mathrm{i}, \mathrm{y}, \mathrm{e}, \varepsilon, \varnothing, \propto, \mathrm{a}, \mathrm{a}, \supset, \mathrm{o}, \mathrm{u} /)^{9}$. Le schéma 2 reconstitue le système des voyelles courtes du suisse romand en s'appuyant sur les travaux de Métral (1977), Schoch (1980), et Schouwey (2008). Il est à noter que, comme toutes les variétés nationales de français (de France, Belgique, Suisse, Canada, et autres), le français appelé suisse romand présente des variations régionales. Ces variations s'expriment principalement à travers la distribution des voyelles : toutes les régions ne s'accordent pas sur la distribution des paires mi-ouvertes / mi-fermées (certaines variétés suisses romandes présentent par exemple une opposition [mo] <maux> vs. [mo] $<$ mot $>$ ), ainsi que sur l'opposition [a]/[a] (Schoch, 1980).

\footnotetext{
${ }^{8}$ Excepté dans un cas où les deux s'étaient préalablement rencontré une fois.

${ }^{9}$ Le statut du schwa étant particulier nous préférons l'exclure de cet inventaire.
} 


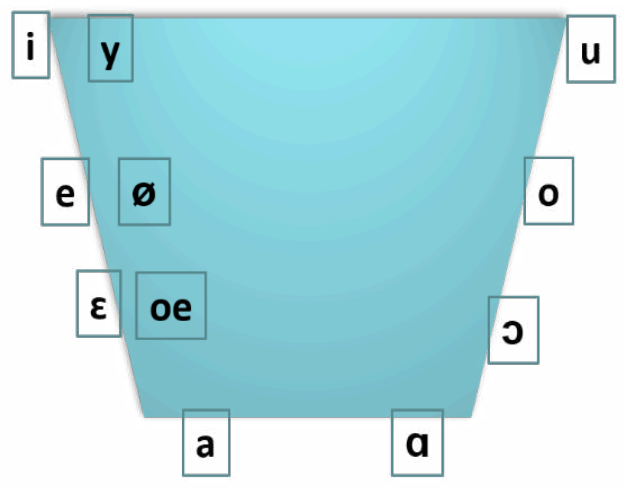

Schéma 2 : Trapèze vocalique des voyelles phonologiques orales courtes du français parlé en Suisse romande

\section{Traitement du corpus}

Notre corpus de travail a bénéficié des transcriptions orthographiques mises à disposition par le projet PFC, suivant ses conventions (Durand et al., 2002). La transcription a été faite sous Praat (Boersma, 2006), chaque groupe de souffle de chaque locuteur et locutrice a été transcrit dans un intervalle distinct. Les passages de parole superposée ont été notés entre chevrons $(<>)$, ce qui a permis de les identifier et de les retirer facilement.

Un repérage des pauses et un redécoupage des tours de parole en unités interpausales a été fait manuellement (le bruit de fond dans les fichiers ne permettant pas de le faire automatiquement), suite à quoi le logiciel SPPAS (Bigi, 2015) a permis un découpage et un alignement en mots, syllabes et phones.

Le grand avantage du logiciel SPPAS, par rapport à d'autres outils plus largement utilisés sur le français, tels que Easy-Align (Goldman, 2011), réside dans le fait qu'il utilise un dictionnaire prenant en compte la variation. Pour aligner le signal et l'annotation phonique, SPPAS se base sur un dictionnaire de correspondance phonie-graphie ; pour la plupart des mots lexicaux de la transcription, plusieurs transcriptions phoniques sont envisagées ; l'algorithme s'appuie ensuite sur les propriétés du signal pour choisir la transcription phonique correspondant le mieux. Ce mode de fonctionnement permet notamment de traiter avec plus de rigueur les variations phoniques comme celles relevant des consonnes de liaison, de la réalisation des schwas ou des réductions de pronoms (ex : [i] ou [il] pour 'il').

\section{Paramètres utilisés pour la caractérisation des changements vocaliques}

Pour caractériser les changements subis par les voyelles dans les quatre styles de parole analysés, nous avons choisi un ensemble de cinq paramètres acoustiques, plusieurs auteurs ayant souligné qu'une combinaison de différentes métriques est plus pertinente pour caractériser les différentes façons dont les systèmes et les catégories vocaliques peuvent varier (voir par exemple Ferguson \& Kewley Port, 2007 ; Harmegnies \& Poch Olivé, 1992).

Nous allons donc décrire la réalisation des voyelles à l'aide des mesures suivantes :

- Leur durée (en secondes);

- Leur moyenne de F1 (en Bark);

- Leur moyenne de F2 (en Bark);

- Leur distance par rapport au centre du système vocalique du locuteur ;

- Leur distance par rapport au centre de la catégorie phonologique à laquelle elles appartiennent ;

- Leur compacité (distance entre les deux premiers formants).

La figure 3 donne un aperçu plus concret de ces paramètres. 


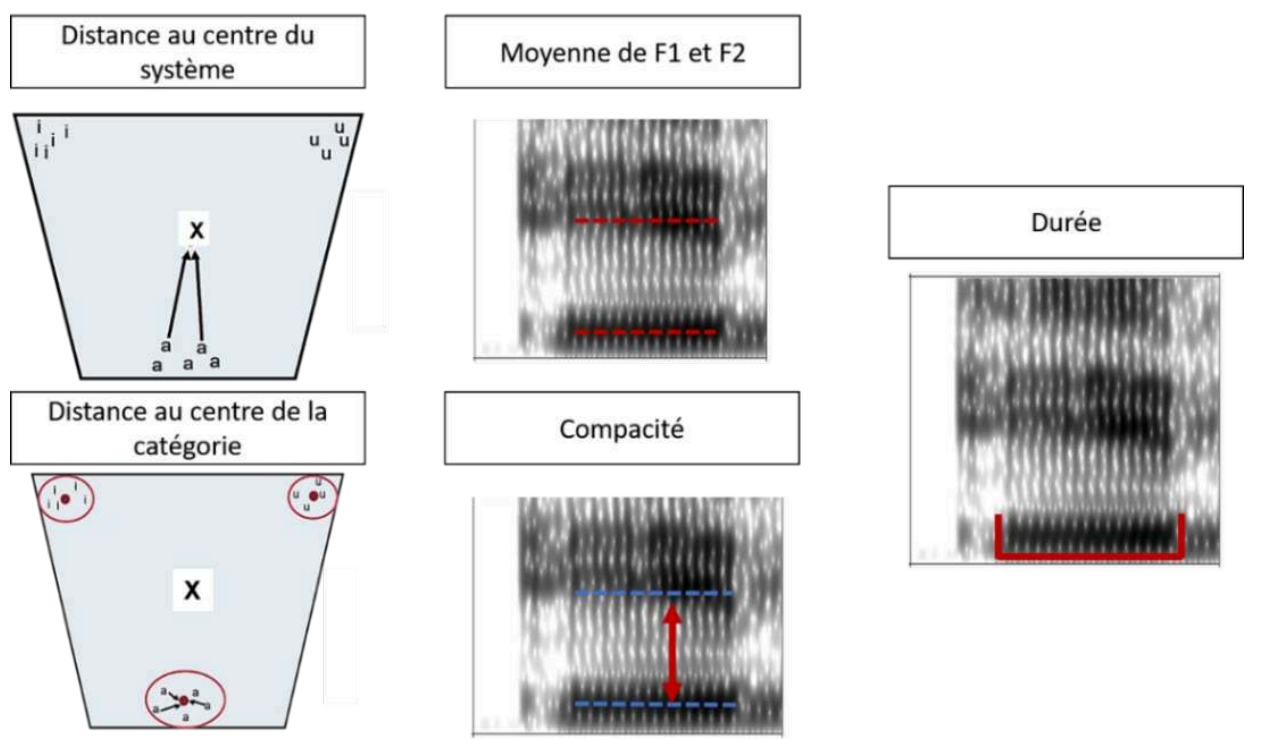

Figure 3. Schématisation des mesures de distance au centre du système, distance au centre de la catégorie, moyennes de F1 et F2, compacité, et durée.

Bien que la variation temporelle soit un domaine complexe qui nécessite la prise en compte de mesures telles que le taux d'articulation, le débit de parole ou le débit articulatoire (Simon et al., 2010), notre étude se concentrera uniquement sur la durée vocalique ${ }^{10}$.

Concernant la variation spectrale, les mesures effectuées ici permettront d'étudier la variation de taille de l'espace acoustique, de la répartition des phones en son sein, ou encore le chevauchement entre ces catégories vocaliques (Fougeron et Audibert, 2011 ; Audibert et al., 2015). Ces informations sont précieuses pour évaluer le degré d'hypoarticulation et observer les modifications dans l'articulation des voyelles autant du point de vue de la quantité de réduction que du type de modifications subies par les phones (par rapport à la cible articulatoire visée). Enfin, nous avons souhaité introduire la mesure de compacité. Cette mesure vise à évaluer l'atteinte de la cible phonétique lors de la phonation (les voyelles diffuses/compactes sont-elles bien réalisées comme telles, ou y a-t-il un phénomène de rapprochement : compaction des diffuses / diffusion des compactes ?). La mesure de compacité nous permettra également d'évaluer la spatialisation de l'articulation des phones en termes d'antériorisation ou de postériorisation.

En partant de l'alignement en phones de notre corpus, un script Praat nous a permis d'extraire la durée de chaque voyelle, ainsi que la moyenne de ses premier et deuxième formants (en Hertz, ensuite convertie en Bark ${ }^{11}$ ). Les mesures de distance au centre du système, au centre des catégories, et la mesure de compacité ont ensuite été calculées à partir des mesures de F1 et F2 en Bark. Ce script a également permis d'extraire le label de chaque exemplaire de voyelle (=sa catégorie vocalique, comme définie par SPPAS), ainsi que son contexte consonantique droit et son contexte consonantique gauche.

\footnotetext{
${ }^{10}$ Des calculs plus pertinents sont en cours (ratio temps de pause/temps de parole, nombre de pause/seconde, nombre de syllabes et phones/seconde, nPvi (Grabe \& Low, 2002) pour saisir les différences de réalisations temporelles et rythmiques de chaque style de parole.

${ }^{11}$ Cette échelle psycho acoustique permet de rendre compte de la variation formantique telle qu'elle est perçue par l'oreille (pour plus de détails voir Nguyen et Adda-Decker, 2013).
} 


\section{Résultats}

\section{Matériel retenu pour l'analyse}

Nous avons extrait de notre corpus 27954 exemplaires de voyelles et leurs caractéristiques en termes de durée et de moyennes de F1 et F2. Après filtrage des valeurs irréalistes (Gendrot \& Adda-Decker, 2005), 25734 exemplaires des voyelles [i, y, e, $\varepsilon$, o, כ, œ, ø, u] ont été conservés (dont 3148 ont été corrigées à la main pour les durées ou les valeurs de formants). L'annotation automatique réalisée par SPPAS ne permettant pas de distinguer les [o] et les [э] (tous deux labélisés $<\mathrm{O} />$ ), les /a/ des /a/ (tous deux labélisés $<\mathrm{A} />$ ), ou les voyelles longues des voyelles courtes, nous ne pourrons pas traiter ces oppositions dans cet article. Le tableau 2 , ci-dessous, donne le nombre d'exemplaires de chaque catégorie vocalique que nous avons retenus dans chaque situation de parole pour effectuer nos analyses.

\begin{tabular}{|c|r|r|r|r|r|}
\hline Style/ Catégorie & \multicolumn{1}{|c|}{ Guidée } & \multicolumn{1}{c|}{ Libre } & \multicolumn{1}{c|}{ Listes } & \multicolumn{1}{c|}{ Texte } & Grand Total \\
\hline$/ \mathbf{d} /$ & 238 & 159 & 531 & 163 & 1091 \\
\hline$/ \mathbf{e} /$ & 810 & 536 & 427 & 524 & 2297 \\
\hline$/ \mathbf{A} /$ & 2486 & 1557 & 834 & 776 & 5653 \\
\hline$/ \mathbf{e} /$ & 2029 & 1007 & 229 & 680 & 3945 \\
\hline$/ \mathbf{\varepsilon} /$ & 1725 & 1014 & 935 & 692 & 4366 \\
\hline$/ \mathbf{i} /$ & 1260 & 785 & 530 & 789 & 3364 \\
\hline$/ \mathbf{O} /$ & 990 & 706 & 319 & 556 & 2571 \\
\hline$/ \mathbf{u} /$ & 552 & 338 & 100 & 254 & 1244 \\
\hline$/ \mathbf{y} /$ & 535 & 310 & 51 & 307 & 1203 \\
\hline Grand Total & $\mathbf{1 0 6 2 5}$ & $\mathbf{6 4 1 2}$ & $\mathbf{3 9 5 6}$ & $\mathbf{4 7 4 1}$ & $\mathbf{2 5 7 3 4}$ \\
\hline
\end{tabular}

Tableau 2. Nombre de voyelles par catégorie et par style pour les 5 couples du corpus suisse romand

\section{Résultats de la comparaison lecture/parole spontanée}

L'analyse statistique des données a été effectuée via un modèle linéaire mixte (Bates et al., 2015), complété par des tests posthoc (Tuckey HSD). Chaque métrique a été définie comme la variable dépendante de son propre modèle. En ce qui concerne les variables indépendantes, le style de parole, le genre du locuteur et la catégorie vocalique ont été définis comme des facteurs fixes, tandis que le contexte consonantique, le locuteur, l'âge, et l'origine régionale ont été définis comme des facteurs aléatoires.

Dans un premier temps nous avons défini deux styles principaux, deux « macro-styles » :

- La lecture : comprenant la lecture de listes de mots et la lecture de texte

- La parole spontanée : comprenant les conversations avec l'enquêtrice et les conversations entre conjoints

L'analyse statistique de ces données a montré un effet significatif du style sur les quatre mesures acoustiques : la durée des phones, leur dispersion au sein du système, leur dispersion au sein de leur catégorie, et leur compacité $(\mathrm{p}<0,001)$. Comme le montre la figure 4 , nos résultats sur le français suisse romand sont congruents avec les résultats de Harmegnies \& PochOlivé (1992), Poch-Olivé \& Harmegnies (1992), Adda-Decker \& Lamel (1999), ou encore Rouas et al. (2010). En parole spontanée, nous observons des durées vocaliques plus courtes, des voyelles moins dispersées au sein du système et plus dispersées au sein de leur catégorie. Ces trois éléments vont tous dans le sens d'un plus fort degré d'hypoarticulation en parole spontanée qu'en lecture. Quant à la mesure de compacité, elle nous indique que les voyelles 
sont légèrement plus compactes, donc plus postérieures, en situation de conversation qu'en situation de lecture. En effet, la proximité de F1 et F2, notamment via un F2 bas, est un trait caractéristique de la postériorité de l'articulation.

Cette analyse ayant principalement pour but de répliquer les résultats déjà mis en évidence par la littérature, nous ne la développerons pas plus. La suite de nos analyses visera à démontrer que lorsque l'on dépasse l'opposition entre les macro-styles « lecture » et « parole spontanée » et que l'on prend en compte les deux conditions de lecture (listes de mots / texte) et la distance sociale entre les interlocuteurs en situation de parole conversationnelle spontanée (enquêtrice / conjoint), l'approche linéaire du continuum hyper-hypo ne suffit plus à expliquer les différences entre styles. Il faut alors passer à une analyse plus fine des combinaisons entre les métriques.
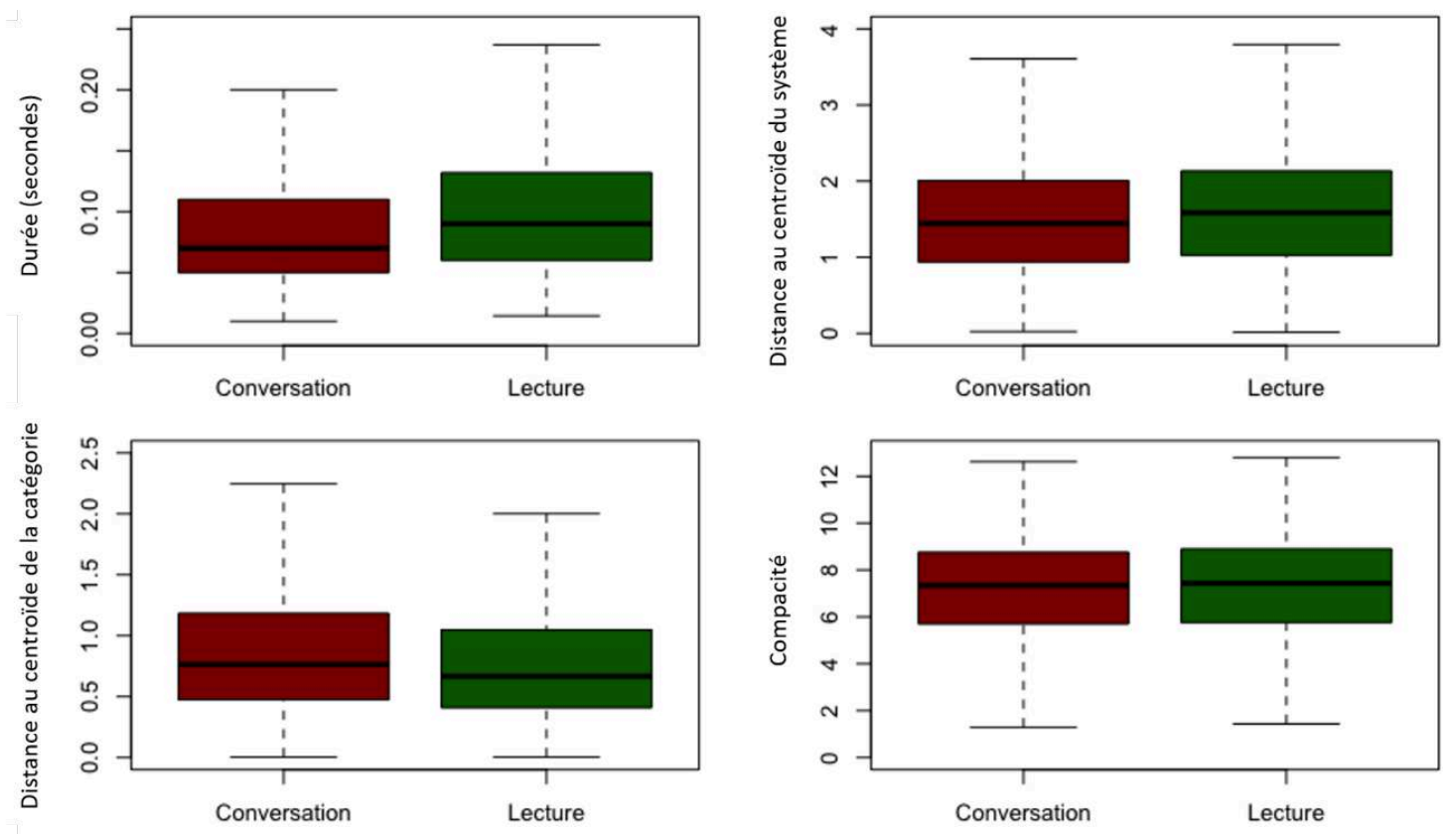

Figure 4. Différences entre les quatre métriques (durée, distance au centroïde du système, distance au centroïde de la catégorie, compacité) en condition de lecture (vert) et de parole spontanée (rouge).

\section{Résultats de la comparaison entre les quatre styles de parole}

De même que pour l'analyse de la différence entre les macro-styles «lecture » et «parole spontanée », l'analyse statistique des données a été effectuée via un modèle linéaire mixte (Bates et al., 2015), complété par des tests posthoc (Tuckey HSD). Chaque métrique a été définie comme la variable dépendante de son propre modèle. En ce qui concerne les variables indépendantes, pour chaque modèle, le style de parole, le genre du locuteur et la catégorie vocalique ont été définis comme des facteurs fixes, tandis que le contexte consonantique, le locuteur, l'âge, et l'origine régionale ont été définis comme des facteurs aléatoires.

Dans cette série d'analyses, nous prenons en compte individuellement les quatre situations de parole, les quatre styles, présentés dans la partie méthodologique, à savoir : la lecture de listes de mots, la lecture de textes, la discussion avec une enquetrice, et la discussion entre conjoints. Le tableau 3 montre les principaux effets mis en évidence par les modèles statistiques. Nous traiterons par la suite plus en détail des résultats concernant les styles de parole, et mentionnerons plus brièvement la variation liée au genre, qui nous parait importante dans la caractérisation des relations entre hypoarticulation et distance sociale. 


\begin{tabular}{|l|l|l|l|l|l|l|l|}
\hline & Style & Locuteur & Genre & Région & Age & $\begin{array}{l}\text { Contexte } \\
\text { Phonémique }\end{array}$ & $\begin{array}{l}\text { Catégorie } \\
\text { Vocalique }\end{array}$ \\
\hline Durée & $* * *$ & $* * *$ & - & - & - & $* * *$ & $* * *$ \\
\hline $\begin{array}{l}\text { Dispersion } \\
\text { Système }\end{array}$ & $* * *$ & $* * *$ & $* * *$ & - & - & $* * *$ & $* * *$ \\
\hline $\begin{array}{l}\text { Dispersion } \\
\text { Catégorie }\end{array}$ & $* *$ & $* * *$ & - & - & - & $* * *$ & $* * *$ \\
\hline Compacité & $* * *$ & $* * *$ & - & - & $* *$ & $* * *$ & $* * *$ \\
\hline
\end{tabular}

Tableau 3 : Récapitulatif des effets principaux du style de parole, du locuteur, de son genre, son origine régionale et son âge, ainsi que du contexte phonémique et de la catégorie vocalique sur les mesures phonétique observées. Les effets non significatifs sont notés, les $* * *$ correspondent à une valeur $\mathbf{p}$ inférieure à 0,001 , les ** correspondent à une valeur $p$ inférieure à 0,01 .

\section{La durée}

Les analyses montrent un effet général du style de parole sur la longueur vocalique $(p<0,001)$. La durée vocalique est beaucoup plus longue pour les voyelles réalisées en lecture de listes de mots $(\mu=135 \mathrm{~ms}, \sigma=77 \mathrm{~ms})$ que pour les voyelles réalisées en situation de conversation $(\mathrm{p}<0,001)$.

On remarque également une différence significative entre la longueur des voyelles lors de la lecture des listes de mots et lors de la lecture du texte $(\mathrm{p}<0,001)$. Dans la condition de lecture de texte, les voyelles sont plus courtes $(\mu=88 \mathrm{~ms}, \sigma=44 \mathrm{~ms})$ qu'en lecture de liste de mots. Il n'est cependant pas à exclure que cette différence soit liée à la différence de structure prosodique entre les deux conditions : en lecture de listes un mot lexical correspond souvent à un mot prosodique (on comptera donc vraisemblablement plus de voyelles accentuées dans cette condition), ce qui n'est pas le cas dans la lecture de texte.

Aucune différence significative de longueur n'est observée entre la lecture de texte et les conditions de conversation.

La conversation avec l'enquêtrice montre des durées vocaliques plus courtes que la discussion entre conjoints, avec une moyenne de $94 \mathrm{~ms}(\sigma=78 \mathrm{~ms})$ pour la discussion avec l'enquêtrice contre $100 \mathrm{~ms}(\sigma=80 \mathrm{~ms})$ pour la discussion entre conjoints $(p<0,001)$ (voir figure 5 ci-dessous). Dans l'hypothèse où la distance sociale impacterait le degré de réduction des voyelles, on s'attendrait à ce que les voyelles soient plus courtes dans la conversation entre conjoints que dans la conversation avec l'enquêtrice. Une hypothèse possible pour expliquer la situation inverse que nous constatons ici (des voyelles plus courtes dans la conversation avec l'enquêtrice) pourrait résider dans la différence d'attitude ou d'engagement des locuteurs et locutrices: une discussion entre conjoints étant porteuse de moins d'enjeux (moins de nouveauté, moins de volonté de paraitre coopératif...) qu'une interaction avec une enquêtrice. Comme le montrent les écarts-types, les durées de ces voyelles varient énormément dans tous les styles, ce qui peut s'expliquer par la grande différence de longueur entre les voyelles accentuées et les voyelles non-accentuées en français (Léon, 1992).

On constate également un effet général du locuteur $(\mathrm{p}<0,001)$, de la catégorie vocalique $(p<0,001)$, et du contexte consonantique $(p<0,001)$, cependant aucun effet de genre, d'âge ou d'origine régionale n'est observé. En somme, si l'on observe effectivement des variations dans la durée en fonction des quatre styles de parole, rien ne montre pour l'instant que l'augmentation de la vitesse d'articulation (que l'on observerait ici à travers des durées vocaliques plus courte) ne soit utilisée comme un indicateur d'une distance sociale plus faible. D'autre part cette variation n'est pas homogène entre les locuteurs ni entre les catégories de 
voyelles, chaque locuteur fait donc varier la durée différemment lors de la production des quatre styles, et ne la fait pas varier de la même façon pour toutes les catégories vocaliques.

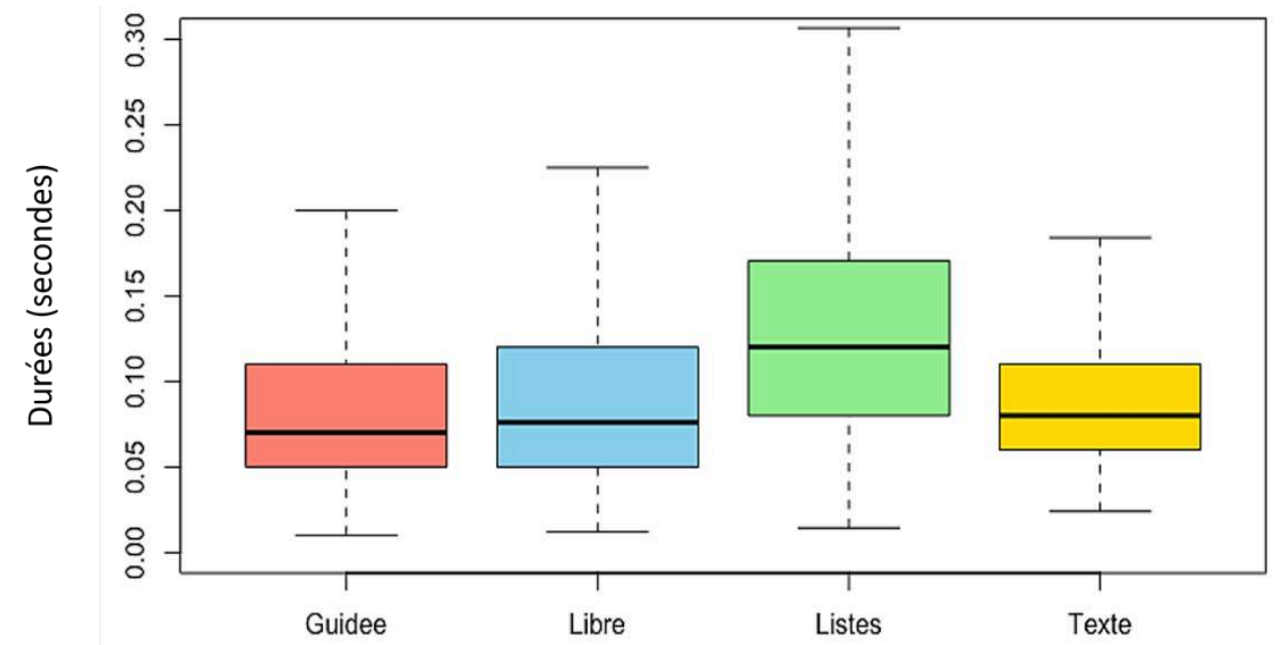

Figure 5 : Représentation de la distribution de la durée vocalique en fonction du style de parole. L'axe des ordonnées donne la durée des voyelles en secondes, et l'axe des abscisses donne le style de parole. Guidée= conversation avec l'enquêtrice ; Libre = conversation avec le ou la conjoint(e).

\section{Les mesures de F1 et F2}

Le tableau A (en annexe) donne à titre indicatif les moyennes des F1 et F2 (Hz, moyenne sur la durée de la voyelle) des différentes catégories vocaliques pour chaque style, en prenant en compte le genre du locuteur. Les mesures statistiques ont été faites sur une conversion en Bark de ces mesures, de façon à être plus proche de la perception auditive humaine.

\section{Les mesures de dispersion des phones au sein du système vocalique}

Concernant les mesures relatives à l'espace occupé par les voyelles, l'aire du système vocalique (obtenue via la fonction poly.area du package PhonR (McCloy, 2016)) est plus vaste en lecture de listes de mots que dans la lecture de texte et les deux styles spontanés conversationnels. L'aire est également plus grande pour la lecture de texte que pour les deux conversations. Enfin, elle est plus vaste lors d'une discussion avec une enquêtrice inconnue que lors d'une discussion entre conjoints. Ces valeurs, résumées dans le tableau 4, indiquent un polygone plus restreint, donc un espace acoustique moins vaste, et des voyelles moins distinctes les unes des autres dans les styles montrant une distance sociale plus faible (conjoint(e) $v s$. enquêtrice) ou un degré de « contrôle $»^{12}$ plus faible (textes vs. listes). À l'inverse, on constate des polygones plus grands, donc des espaces acoustiques plus vastes, et des voyelles plus distinctes les unes des autres dans les situations où la distance sociale est plus forte (enquêtrice $v s$. conjoint(e)) ou dans les situations où le degré de contrôle est plus fort (listes vs. textes). Ces résultats sont interprétables comme une plus forte hypoarticulation lorsque la distance sociale est moindre et lorsque le degré de contrôle est moindre, et une plus forte hyperarticulation lorsque la distance sociale est forte et lorsque le degré de contrôle est fort.

\footnotetext{
${ }^{12}$ Nous reprenons ici la distinction entre les styles «contrôlés » et les styles « spontanés » utilisée par Yaeger (1974) dans une situation expérimentale similaire à la nôtre, pour rendre compte des différences entre les styles impliquant la lecture de textes ou de mots (contrôlés) et les styles impliquant de la parole spontanée (spontanés).
} 


\begin{tabular}{|l|l|l|l|l|}
\hline & Listes & Texte & Guidée & Libre \\
\hline Aire du polygone & 38592.78 & 33600.37 & 21917.37 & 13043.52 \\
\hline
\end{tabular}

Tableau 4 : Mesures de l'aire des polygones constitués par l'espace occupé par les voyelles dans les différents styles de parole

Les analyses montrent un effet général du style, du locuteur, du genre, de la catégorie vocalique, et du contexte phonémique $(\mathrm{p}<0,001)$ sur la distance des voyelles par rapport au centroïde (calculé pour chaque locuteur) du système vocalique.

Comme montré en figure 6, la taille de l'espace occupé par le système vocalique est plus grande pour la lecture de mots que pour les deux situations de conversation $(p<0.001)$, l'espace est également plus vaste pour la discussion avec une enquêtrice inconnue que pour la discussion entre conjoints $(\mathrm{p}<0,001)$.

La dispersion au sein du système des voyelles produites en condition de lecture de texte montre, elle, un schéma assez singulier : l'espace acoustique est plus vaste en lecture de texte que dans la condition de discussion entre conjoints ( $\mathrm{p}<0,01)$, mais n'apparait pas comme étant significativement plus vaste ou plus restreint que l'espace acoustique occupé par les voyelles en lecture de listes ou en conversation avec l'enquêtrice.

Aucun impact de l'âge ou de l'origine régionale (Martigny ou Neuchâtel) n'est à noter.

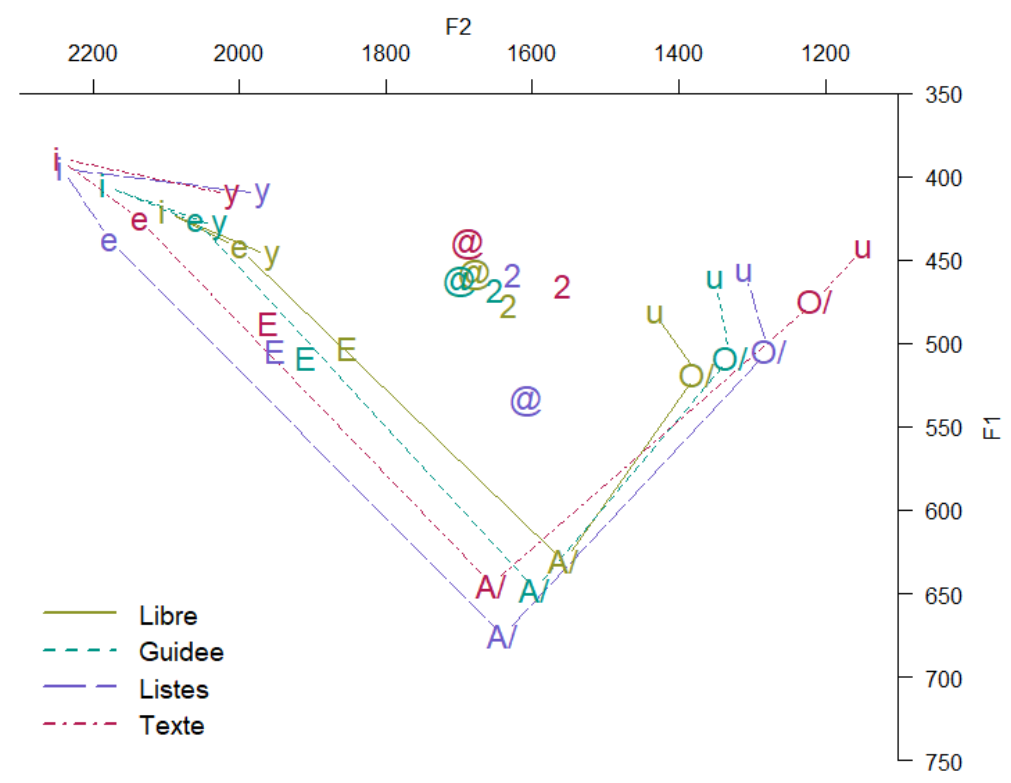

Figure 6 : Représentations de l'espace occupé par les voyelles dans chaque style de parole. L'axe des ordonnées donne les valeurs de F1 en Hertz, et l'axe des abscisses donne les valeurs de F2 en Hertz.

L'effet général du genre $(\mathrm{p}<0,001)$ montre que la dispersion au sein du système des voyelles produites par les femmes est, de façon générale, plus vaste que celle observée chez les hommes. L'interaction entre le genre et le style de parole a été rajoutée au modèle linéaire mixte, et apparait comme ayant un impact significatif sur la dispersion au sein du système $(p<0,001)$. Les tests posthoc réalisés par la suite ont montré que la dispersion au sein du système des voyelles produites par les femmes était significativement plus vaste que la dispersion au sein du système des voyelles produites par les hommes en conversation entre conjoints $(p<0,01)$ et en lecture de listes de mots $(\mathrm{p}<0,001)$ uniquement (visible sur la figure 7 et le tableau 5 , cidessous). Ce fait nouveau nous renseigne sur une asymétrie entre les genres dans l'expression de la distance sociale : si la réduction des phones est, comme le suggère notre hypothèse, liée à une plus faible distance sociale entre les locuteurs, alors il s'avère que les hommes sont plus enclins à exprimer cette proximité que les femmes. Nous reviendrons sur cette hypothèse dans la discussion. 

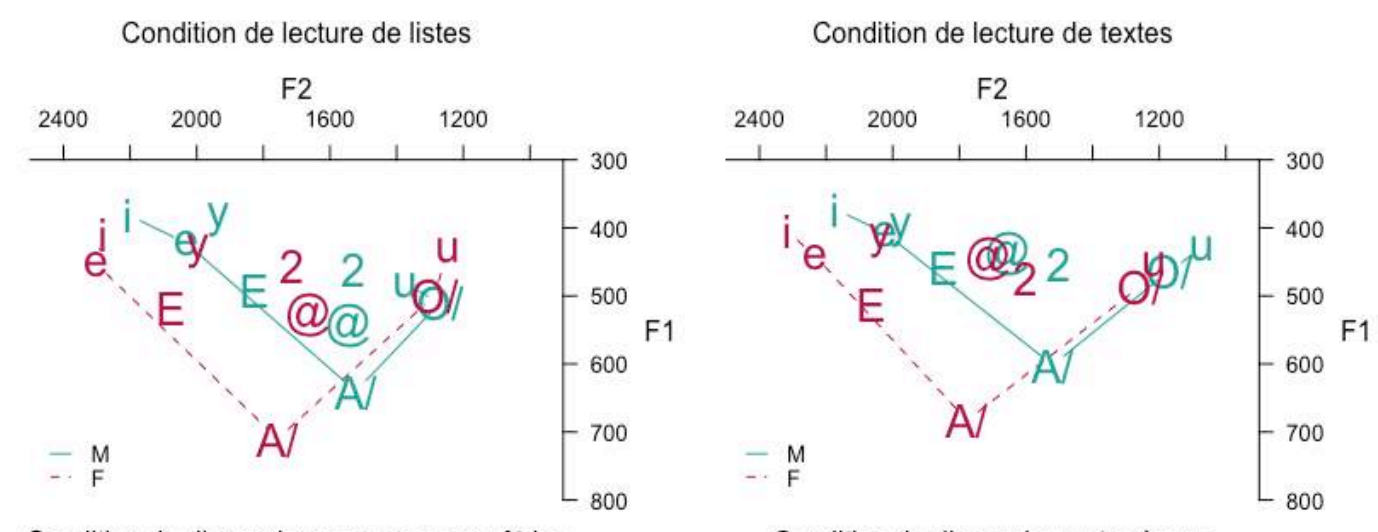

Condition de discussion avec une enquêtrice

Condition de discussion entre époux
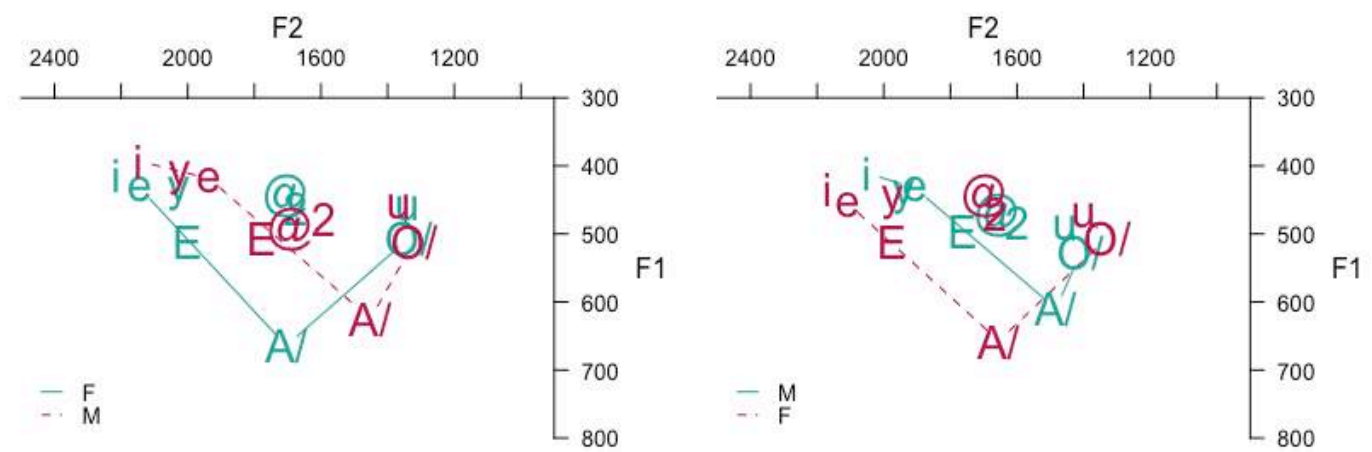

Figure 7 : Représentations de l'espace occupé par les voyelles dans chaque style de parole en fonction du genre. L'axe des ordonnées donne les valeurs de F1 en Hertz, et l'axe des abscisses donne les valeurs de F2 en Hertz.

\section{Les mesures de dispersion des phones au sein de leurs catégories vocaliques}

En ce qui concerne la distance au centre de la catégorie, interprétable comme la dispersion intracatégorie des phones, soit l'espace acoustique occupé par une catégorie vocalique au sein du système, on note un effet général du style $(\mathrm{p}<0,01)$, du locuteur, du contexte phonémique, et de la catégorie vocalique $(\mathrm{p}<0,001)$. Aucun effet de l'âge, du genre ou de l'origine régionale n'est constaté.

La lecture de texte montre la dispersion intracatégorie la moins grande. En ce qui concerne les trois autres conditions (lecture de listes de mots, conversation avec une enquêtrice et conversation entre conjoints) la différence est ténue, même si la lecture de listes de mots semble montrer une plus grande dispersion intracatégorie que les situations de conversation. On ne remarque cependant pas de différences de dispersion intracatégorie des phones entre les deux types de conversations.

Ces résultats nous incitent à penser que cette mesure peut être pertinente pour la caractérisation des différents styles de parole (comme montré plus haut dans les différences entre les macro styles «lecture » et «parole spontanée»), mais qu'elle ne l'est pas pour l'expression de la distance sociale dans les situations de conversation.

\section{Les mesures de compacité des voyelles}

L'examen de la variation de compacité des voyelles a montré un effet général du style, du locuteur, de la catégorie vocalique, et du contexte consonantique $(\mathrm{p}<0,001)$. Cependant les tests posthoc ont montré que ces différences de compacité des voyelles n'étaient en fait significatives qu'entre la lecture de texte et la lecture de mots, la lecture de texte et la discussion entre conjoints, la lecture de texte et la discussion avec l'enquêtrice, et finalement la discussion entre conjoints et la discussion avec l'enquêtrice. Aucune différence significative de compacité des 
voyelles n'est constatée entre la lecture de listes de mots et la discussion entre conjoints, ni entre la lecture de listes de mots et la discussion avec l'enquêtrice. Nous supposons que cette absence de significativité est due au peu de données dans la condition de lecture de mots.

En ce qui concerne les deux situations de conversation, comme le montre la figure 8 , les conversations entre conjoints montrent des voyelles plus compactes que les conversations avec une enquêtrice $(p<0,01)$, ce qui nous amène à penser que la postériorisation de l'articulation des phones pourrait révéler une distance sociale plus faible entre les locuteurs. Cette hypothèse est cohérente avec les résultats observés pour la lecture de texte, situation plus contrôlée, qui montre également des voyelles plus diffuses que les deux types de conversations $(p<0.001)$.

La différence entre la lecture de texte et la lecture de liste nous parait être liée à un déséquilibre dans la répartition des phones (plus de voyelles compactes en lecture de listes qu'en lecture de textes, voir tableau 2).

On remarque également un effet, plus surprenant, de l'âge, avec des voyelles plus postérieures chez les plus de 50 ans. Nous ne développerons pas ce point ici, cependant nous émettons deux hypothèses : 1) un changement d'ordre diachronique s'est opéré, et l'articulation des phones se fait plus à l'avant de la cavité buccale chez les nouvelles générations que chez les anciennes ; 2) le vieillissement entraine des modifications dans l'articulation des phones, notamment sur le plan de la compacité (comme semblent le suggérer D'Alessandro et Fougeron, 2017).

Cette différence liée à l'âge, ainsi que celle liée au genre mise en évidence pour la dispersion des phones au sein du système, montre qu'il est nécessaire d'observer les réalisations d'un plus grand éventail de locuteurs et locutrices, pour déterminer en quoi leurs caractéristiques individuelles modifient la façon dont ils s'adressent à un proche ou à une enquêtrice.

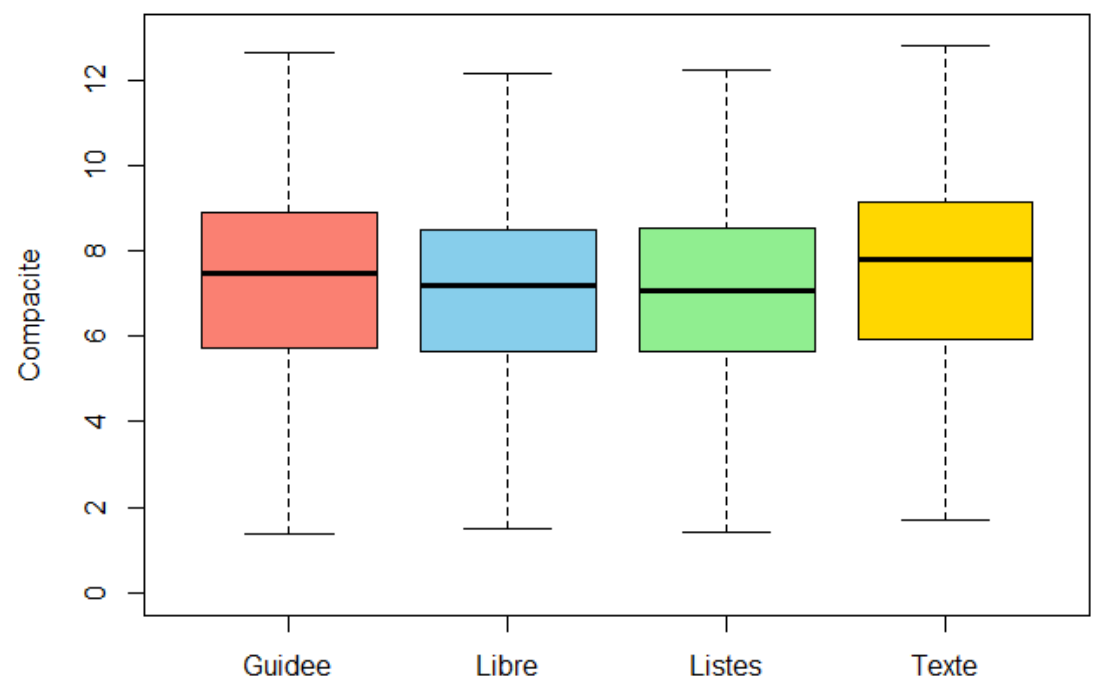

Figure 8 : Répartition des mesures de compacité (ordonnées) en fonction du style de parole (abscisses). Plus l'indice de compacité est élevé, plus les voyelles sont diffuses (=antérieures).

\section{Conclusion et discussion}

Nos résultats montrent une utilisation différente des métriques phonétiques en fonction des styles de parole. S'il a déjà été démontré que les voyelles sont généralement plus courtes et plus centralisées en parole conversationnelle qu'en lecture (Harmegnies \& Poch-Olivé, 1992 ; PochOlivé \& Harmegnies, 1992 ; Rouas et al., 2010 ; entre autres), notre étude apporte une pierre supplémentaire dans l'étude de la variation et de la réduction des phones (hypoarticulation) dans les différents styles de parole. Nous avons démontré deux éléments principaux : 1) pour 
étudier en détail les différences phoniques entre les styles de parole, il ne suffit pas de leur attribuer un degré d'hyper- ou hypo- articulation, encore moins de créer une dichotomie entre des styles hyperarticulés et des styles hypoarticulés. Il est nécessaire de prendre en compte la façon dont se combinent les différents aspects phonétiques de la variation : les mesures utilisées pour caractériser la variation ou la réduction vocalique ne sont pas nécessairement toutes pertinentes pour la description de chaque style, ni toutes corrélées au sein de chaque style. 2) en situation de conversation, le degré de réduction des voyelles est corrélé au degré de distance sociale entre les locuteurs, cette corrélation entre la réduction vocalique et la proximité sociale est visible à travers les mesures de durée vocalique, de dispersion au sein du système, et de compacité.

Dans notre étude, la situation la plus contrôlée, à savoir la lecture de listes de mots, montre les voyelles les plus longues, les plus dispersées au sein du système, les plus dispersées au sein de leur catégorie, et les plus compactes (donc plus postérieures). Dans une situation de lecture de texte, toujours contrôlée mais moins artificielle, et donc supposément moins soignée, les voyelles sont légèrement moins longues, moins dispersées au sein du système, beaucoup moins dispersées au sein de leur catégorie, et plus diffuses. Nous avons donc ici affaire à deux types de lecture différents, ayant des caractéristiques propres sur le plan de la réduction vocalique et de la compacité ${ }^{13}$. Cependant, lorsque l'on observe la façon dont les deux conditions de lecture diffèrent des conditions de conversation spontanée, on remarque une hétérogénéité des patterns de variation : la différence entre la lecture de listes et les styles spontanés conversationnels ne s'exprime pas par les mêmes métriques que la différence entre la lecture de texte et les styles spontanés conversationnels.

Pour les deux conditions de conversation, les voyelles sont plus courtes, plus centralisées, moins dispersées au sein de leur catégorie, et plus diffuses que dans la condition de lecture de listes de mots ; cependant elles sont moins diffuses et plus dispersées au sein de leur catégorie que dans la lecture de textes. C'est ce pattern qui nous incite à penser qu'il est préférable de prendre en compte la combinaison des caractéristiques phoniques au sein de chaque style, et non pas uniquement le placement de ces styles sur le continuum de l'hyper-hypoarticulation. On remarque également que la conversation avec une enquêtrice montre des voyelles plus dispersées au sein du système, et plus diffuses que dans les conversations entre conjoints, mais légèrement moins longues. En somme les voyelles sont spectralement plus réduites (mais pas temporellement plus réduites), le degré d'hypoarticulation est plus fort lorsque l'on s'adresse à un proche. Ce fait vient corroborer notre hypothèse concernant la distance sociale : la réduction spectrale des voyelles est corrélée à la proximité sociale entre les participants et participantes de l'interaction. Cependant, encore une fois, ce n'est pas une simple question de placement sur un continuum hypo-hyper, puisque toutes nos mesures ne vont pas dans le même sens : la durée des phones montre moins de réduction temporelle en situation de conversation entre conjoints qu'en interaction avec une enquêtrice inconnue (alors qu'on s'attendrait au contraire).

Pour conclure sur la variation phonostylistique, bien que l'on constate une plus grande réduction vocalique dans les situations de parole spontanée conversationnelle que dans les situations de lecture, il semble que la description du phénomène soit plus complexe qu'une

\footnotetext{
${ }^{13}$ La compacité donne des informations plus complexes à analyser que les autres mesures : le style dans lequel la parole est supposée être la plus surveillée (lecture de listes de mots) montre les voyelles les plus compactes, cependant l'autre style supposé montrer une articulation plus soignée (la lecture de texte) montre les voyelles les plus diffuses. Quant aux conditions de conversation, la discussion avec un inconnu montre des voyelles plus diffuses que la discussion entre conjoints. Si l'on examine qualitativement les mesures de F1 et F2 (voir tableau A en annexe), on remarque que la variation se fait plus sur l'axe de l'antériorité/postériorité (F2), ce qui pourrait indiquer que la mesure de compacité (donnant des informations sur l'antériorité/postériorité des voyelles) serait pertinente pour l'analyse de la variation phonostylistique, bien qu'ici les résultats liés à ces mesures restent obscurs.
} 
simple augmentation du degré d'hypoarticulation : chaque style mobilise les caractéristiques temporelles et spectrales des voyelles différemment. Le tableau 6 montre l'utilisation des métriques faite pour chaque style.

\begin{tabular}{|c|c|c|c|c|}
\hline & Durée & $\begin{array}{c}\text { Distance au } \\
\text { centroïde du } \\
\text { système }\end{array}$ & $\begin{array}{c}\text { Distance au } \\
\text { centroïde de la } \\
\text { catégorie }\end{array}$ & Compacité \\
\hline Lecture de listes & ++++ & ++++ & ++ & ++++ \\
\hline $\begin{array}{c}\text { Lecture de } \\
\text { textes }\end{array}$ & +++ & +++ & + & + \\
\hline $\begin{array}{c}\text { Conversation } \\
\text { EQ }\end{array}$ & + & ++ & ++ & ++ \\
\hline $\begin{array}{c}\text { Conversation } \\
\text { Epoux }\end{array}$ & ++ & + & ++ & +++ \\
\hline
\end{tabular}

Tableau 5 : Récapitulatif des caractéristiques phonétiques de chaque style de parole étudié. Le nombre de «+» détermine le degré de la variable (ex : durée*lecture de listes : «++++» car sur les quatre styles la lecture de listes montre les voyelles les plus longues).

Les situations les moins contrôlées, ainsi que celles pour lesquelles la distance sociale est moins forte, montrent bien des traces de plus forte hypoarticulation à travers la réduction de la durée vocalique ou un espace acoustique plus réduit, notamment. Cependant il apparait important de prendre en compte la combinaison de métriques observée dans chaque style, aucun ne montrant la même utilisation des quatre métriques. Cette approche nouvelle de l'étude de la variation et de la réduction vocalique permet de mettre en évidence les caractéristiques phonétiques de chaque style de parole de façon plus précise. Elle nous permet également de mettre en évidence l'importance de la distance sociale entre les interlocuteurs dans la production de la parole, qui, bien qu'elle soit considérée dans les études interactionnistes depuis près d'un demi-siècle (voir Brown \& Fraser, 1979), n'était jusqu'alors que très peu détaillée, ou réduite à une distinction de formalité ou de contrôle, par les travaux étudiant les styles de parole d'un point de vue phonétique.

\section{Remerciements}

Nous souhaitons remercier la Professeure Isabelle Racine (Université de Genève) pour la mise à disposition des données ainsi que tou'te's contributeur'trice's qui ont permis la constitution de ce corpus. Enfin nous remercions Maria Candea et Marie-Hélène Côté pour leur relecture et leurs remarques précieuses sur nos premières analyses et interprétations.

\section{Bibliographie}

ADDA-DECKER Martine \& LAMEL Lori, 1999, «Pronunciation variants across system configuration, language and speaking style », Speech Communication, vol. 29, no 2-4, 83-98.

ANDREASSEN Helene, MAITRE Raphaël, RACINE Isabelle, 2010, " La Suisse », dans : Detey, S., Durand J., Laks B., Lyche C., (eds) : Les variétés du français parlé dans l'espace francophone : ressources pour l'enseignement, Paris : Ophrys, 211-233.

ANDREASSEN Helene, \& RACINE Isabelle, 2016, « Variation in Switzerland: the behaviour of schwa in Martigny, Neuchâtel and Nyon », In S. Detey, J. Durand, B. Laks et C. Lyche (eds), Varieties of Spoken French, Oxford : Oxford University Press, 430-440. 
AUDIBERT Nicolas, FOUGERON Cécile, GENDROT Cédric, et ADDA-DECKER Martine, 2015, « Duration-vs. style-dependent vowel variation : a multiparametric investigation. ", In $18^{\text {th }}$ International Congress of Phonetic Sciences (ICPhS'15).

BATES Douglas, MAECHLER Martin, BOLKER Ben, WALKER Steven, CHRISTENSEN Rune, HAUBO Bojesen, SINGMANN Henrik, DAI Bin \& GROTHENDIECK Gabor, 2015, « Package 'lme4' ", Convergence, vol. 12, no 1.

BECKER Howard, \& USEEM Ruth Hill, 1942, « Sociological analysis of the dyad », American Sociological Review, 7(1), 13-26.

BIGI Brigitte, 2015, "SPPAS-multi-lingual approaches to the automatic annotation of speech », The Phonetician, 111(ISSN: 0741-6164), 54-69.

BOERSMA Paul, 2002, "Praat, a system for doing phonetics by computer ", Glot international, vol. 5.

BROWN Penelope \& FRASER Collin, 1979, «Speech as a marker of situation », In Social markers in speech, Cambridge University Press, 33-62.

D'ALESSANDRO Daria \& FOUGERON Cécile, 2018, «Réduction de la coarticulation et vieillissement ", Dans les actes des 32emes Journées d'Etude sur la Parole (Aix-enProvence).

DEVILLA Lorenzo \& TRIMAILLE Cyril, 2010, « Variantes palatalisées/affriquées en français hexagonal : quel(s) statut(s) sociolinguistique(s) pour quel destin ? », In M. Iliescu, H. Siller-Runggaldier \& P. Danler (eds.), Actes $d u 25^{e}$ congrès international de linguistique et philologie romanes (vol. 4), Berlin : Mouton de Gruyter, 99-108.

DURAND Jacques, LAKS Bernard, \& LYCHE Chantal, 2002, « Synopsis du projet PFC, La phonologie du Français contemporain : Usages, Variétés et Structure », Bulletin pfc, vol. 1, p. 5-7.

ESKENAZI Maxine, 1993, «Trends in speaking styles research », In $3^{\text {rd }}$ European Conference on Speech Communication and Technology.

FERGUSON Sarah Hargus \& KEWLEY-PORT Diane, 2007, « Talker differences in clear and conversational speech: Acoustic characteristics of vowels », Journal of Speech, Language, and Hearing Research, 50(5) : 1241-1255.

FONAGY Ivan, 1983, La vive voix : essais de psycho-phonétique, volume 20. Payot.

FOUGERON Cécile, 2005, "Introduction à la Phonologie Articulatoire », In J. Durand, V. Rey, S. Wauquier-Gravelines and N. Nguyen, Phonologie et Phonétique : Approches contemporaines, Hermes.

FOUGERON Cécile \& AUDIBERT Nicolas, 2011, «Testing various metrics for the description of vowel distortion in dysarthria », Actes de ICPhS, 687-690.

GAHL Susanne, 2015, « Lexical competition in vowel articulation revisited: Vowel dispersion in the easy/hard database » Journal of Phonetics, 49, 96-116.

GENDROT Cédric \& ADDA-DECKER Martine, 2005, « Impact of duration on f1/f2 formant values of oral vowels: an automatic analysis of large broadcast news corpora in French and German », Variations, 2(22.5), 2-4.

GOLDMAN Jean-Philippe, 2011, «EasyAlign: an automatic phonetic alignment tool under Praat », In the proceedings of InterSpeech, Florence (Italy).

GRABE Esther \& LOW Ee Ling, 2002, « Durational variability in speech and the rhythm class hypothesis ", Papers in laboratory phonology, vol. 7, n515-546.

HARMEGNIES Bernard \& POCH-OLIVÉ Dolors, 1994, « Formants frequencies variability in French vowels under the effect of various speaking styles ", Le Journal de Physique IV, 4(C5):C5-509.

KOPPEN Kim, ERNESTUS Mirjam \& VAN MULKEN Margot, 2017, « The influence of social distance on speech behavior: Formality variation in casual speech », Corpus Linguistics and Linguistic Theory, 15(1), 139-165. 
LABOV William, 1972, Sociolinguistic patterns, Number 4, University of Pennsylvania Press. LABOV William, 2002, "Driving forces in linguistic change », In International Conference on Korean Linguistics, Seoul National University, Seoul, South Korea.

LABOV William, 2006 ( $2^{\mathrm{e}}$ ed.), The social stratification of English in New York city. Cambridge University Press.

LINDBLOM Bjorn, 1963, "Spectrographic study of vowel reduction », In The journal of the Acoustical society of America, 35(11),1773-1781.

LINDBLOM Björn \& SUNDBERG Johan, 1971, «Acoustical consequences of lip, tongue, jaw, and larynx movement », The Journal of the Acoustical Society of America, vol. 50, n 4 B, p. 1166-1179.

LINDBLOM Bjorn, 1990, «Explaining phonetic variation: A sketch of the h\&h theory », In Speech production and speech modelling, pages 403-439, Springer.

LYCHE Chantal, 2010, " Le français de référence : éléments de synthèse », Detey S. et al., Les variétés du français parlé dans l'espace francophone : ressources pour l'enseignement, Ophrys, Paris, 143-165.

MATHON Catherine, 2014, «Perception des phonostyles et représentativité du phonogenre : le cas du commentaire sportif en direct », Nouveaux cahiers de linguistique française, vol. 31, p. 93-103.

MÉTRAL Jean-Pierre, 1977, « Le vocalisme du français en Suisse romande Considérations phonologiques », Cahiers Ferdinand de Saussure, n³1, 145-176.

MCCLOY Daniel, 2016, PhonR: tools for phoneticians and phonologists, R package version $.0-7$.

POCH-OLIVE Dolors \& HARMEGNIES Bernard, 1992, «Variations structurelles des systèmes vocaliques en français et espagnol sous l'effet du style de parole », Le Journal de Physique IV, 2(C1) : C1-283.

RACINE Isabelle \& ANDREASSEN Helene, 2012, «A phonological study of a Swiss French variety », dans Phonological variation in French: Illustrations from three continents, 11, p.173.

ROUAS Jean-Luc, BEPPU Mayumi \& ADDA-DECKER Martine, 2010, "Comparison of spectral properties of read, prepared and casual speech in French », In $L R E C$.

SCARBOROUGH Rebecca \& ZELLOU Georgia, 2013, " Clarity in communication: "clear" speech authenticity and lexical neighborhood density effects in speech production and perception », The Journal of the Acoustical Society of America, n¹34(5), 3793-3807.

SCARBOROUGH Rebecca, DMITRIEVA Olga, HALL-LEW Lauren, ZHAO Yuan \& BRENIER Jason, 2007, «An acoustic study of real and imagined foreigner-directed speech », Journal of the Acoustical Society of America, 121(5): 3044.

SCARBOROUGH Rebecca, 2013, "Neighborhood-conditioned patterns in phonetic detail: Relating coarticulation and hyperarticulation », Journal of Phonetics, 41(6), 491-508.

SCHOCH Marianne, FURRER Otto \& LAHUSEN Thomas, 1980, "Résultats d'une enquête phonologique en Suisse romande », Bulletin de la Section de linguistique de la Faculté des lettres de Lausanne, $\mathrm{n}^{\circ} 2,1-38$.

SCHOUWEY Vanessa, 2008, Les variantes cantonales dans la prononciation des voyelles du français en Suisse romande, Thèse de doctorat, Université de Genève.

SIMON Anne-Catherine, AUCHLIN Antoine, AVANZI Mathieu, GOLDMAN Jean-Philippe, 2010, «Les phonostyles : une description prosodique des styles de parole en français » dans Les voix des Français, (Vol.2) M. Abecassis \& G. Ledegen, Berne, Peter Lang.

TRIMAILLE Cyril, CANDEA Maria, et LEHKA-LEMARCHAND Iryna, 2012, « Existe-t-il une signification sociale stable et univoque de la palatalisation/affrication en français ? Étude sur la perception de variantes non standard », dans SHS Web of Conferences (Vol. 1, pp. 2249-2262), EDP Sciences. 
TRUDGILL Peter \& TRUDGILL Stephen, 1974, The social differentiation of English in Norwich (Vol. 13), CUP Archive.

VERNET Marie \& TRIMAILLE Cyril, 2007, « Contribution à 1'analyse de la palatalisation en français parle contemporain. ", Nottingham French Studies, n46(2), 82-99.

WOEHRLING Cécile, 2009, Accents régionaux en français: perception, analyse et modélisation à partir de grands corpus, Thèse de Doctorat de l’Université Paris Sud.

YAEGER Malcah, 1974, Speaking Syles: Some Phonetic Realizations and their Significance, Pennsylvania Working Papers on Linguistic Change and Variation, Vol. 1, Nº1. 1.

ZELLOU Georgia \& SCARBOROUGH Rebecca, 2015, « Lexically conditioned phonetic variation in motherese: age-of-acquisition and other word-specific factors in infant-and adult-directed speech », Laboratory Phonology, n6(3-4), 305-336.

\section{Annexe}

\begin{tabular}{|c|c|c|c|c|c|c|c|c|c|c|c|c|c|c|c|c|}
\hline & \multicolumn{4}{|c|}{ Guidée } & \multicolumn{4}{|c|}{ Libre } & \multicolumn{4}{|c|}{ Liste } & \multicolumn{4}{|c|}{ Texte } \\
\hline & $\mathbf{F}$ & & $\mathbf{M}$ & & $\mathbf{F}$ & & M & & $\mathbf{F}$ & & $\mathbf{M}$ & & $\mathbf{F}$ & & M & \\
\hline & $\begin{array}{c}\text { F1 } \\
\text { (Hz) }\end{array}$ & $\begin{array}{c}\text { F2 } \\
\text { (Hz) }\end{array}$ & $\begin{array}{c}\text { F1 } \\
\text { (Hz) }\end{array}$ & $\begin{array}{c}\text { F2 } \\
\text { (Hz) }\end{array}$ & $\begin{array}{c}\text { F1 } \\
\text { (Hz) }\end{array}$ & $\begin{array}{c}\text { F2 } \\
\text { (Hz) }\end{array}$ & $\begin{array}{c}\text { F1 } \\
\text { (Hz) }\end{array}$ & $\begin{array}{c}\text { F2 } \\
\text { (Hz) }\end{array}$ & $\begin{array}{c}\text { F1 } \\
\text { (Hz) }\end{array}$ & $\begin{array}{c}\text { F2 } \\
\text { (Hz) }\end{array}$ & $\begin{array}{c}\text { F1 } \\
(\mathrm{Hz})\end{array}$ & $\begin{array}{c}\text { F2 } \\
\text { (Hz) }\end{array}$ & $\begin{array}{c}\text { F1 } \\
(\mathrm{Hz})\end{array}$ & $\begin{array}{c}\text { F2 } \\
(\mathrm{Hz})\end{array}$ & $\begin{array}{c}\text { F1 } \\
\text { (Hz) }\end{array}$ & $\begin{array}{c}\text { F2 } \\
\text { (Hz) }\end{array}$ \\
\hline $\begin{array}{l}1 \varnothing \\
1\end{array}$ & 462 & 1676 & 477 & 1592 & 470 & 1664 & 482 & 1600 & 455 & 1717 & 461 & 1541 & 473 & 1602 & 454 & 1502 \\
\hline $\begin{array}{l}/ \propto \\
/\end{array}$ & 443 & 1705 & 491 & 1691 & 441 & 1699 & 471 & 1660 & 524 & 1658 & 542 & 1561 & 443 & 1712 & 435 & 1655 \\
\hline$/ a /$ & 664 & 1704 & 624 & 1451 & 657 & 1654 & 610 & 1482 & 707 & 1762 & 648 & 1535 & 683 & 1776 & 601 & 1519 \\
\hline$/ \mathrm{e} /$ & 433 & 2143 & 418 & 1935 & 455 & 2108 & 432 & 1905 & 452 & 2307 & 424 & 2040 & 441 & 2232 & 407 & 2023 \\
\hline$/ \varepsilon /$ & 510 & 2001 & 504 & 1777 & 511 & 1976 & 496 & 1763 & 516 & 2092 & 494 & 1835 & 513 & 2063 & 458 & 1846 \\
\hline /i/ & 414 & 2213 & 393 & 2146 & 436 & 2155 & 411 & 2049 & 409 & 2285 & 382 & 2210 & 404 & 2317 & 372 & 2174 \\
\hline $\begin{array}{l}\text { /0 } \\
/\end{array}$ & 506 & 1336 & 510 & 1319 & 505 & 1328 & 527 & 1408 & 501 & 1298 & 506 & 1259 & 485 & 1255 & 462 & 1168 \\
\hline $\begin{array}{l}/ \mathbf{u} \\
/\end{array}$ & 461 & 1341 & 459 & 1365 & 471 & 1399 & 490 & 1457 & 428 & 1218 & 479 & 1382 & 453 & 1216 & 430 & 1072 \\
\hline$/ y /$ & 439 & 2027 & 417 & 2025 & 453 & 1973 & 444 & 1941 & 439 & 2006 & 388 & 1937 & 419 & 2036 & 402 & 1976 \\
\hline
\end{tabular}

Tableau A : Valeurs moyennes des F1 et F2 (Hz) des voyelles du français suisse romand dans trois styles de parole et en fonction du genre du locuteur 


\section{GLOTTOPOL}

Revue de sociolinguistique en ligne

Comité de rédaction : Michaël Abecassis, Salih Akin, Sophie Babault, Claude Caitucoli, Véronique Castellotti, Régine Delamotte, Robert Fournier, Stéphanie Galligani, Emmanuelle Huver, Normand Labrie, Foued Laroussi, Benoit Leblanc, Fabienne Leconte, Gudrun Ledegen, Danièle Moore, Clara Mortamet, Alioune Ndao, Isabelle Pierozak, Gisèle Prignitz.

Rédactrice en chef : Clara Mortamet.

Comité scientifique : Claudine Bavoux, Michel Beniamino, Jacqueline Billiez, Philippe Blanchet, Pierre Bouchard, Ahmed Boukous, Pierre Dumont, Jean-Michel Eloy, Françoise Gadet, Monica Heller, Caroline Juilliard, Jean-Marie Klinkenberg, Jean Le Du, Marinette Matthey, Jacques Maurais, Marie-Louise Moreau, Robert Nicolaï, Didier de Robillard, Paul Siblot, Claude Truchot, Daniel Véronique.

\section{Comité de lecture pour ce numéro :}

Mickael Abecassis, Michelle Auzanneau, Salih Akin, Céline Amourette, Gabriel Bergounioux, Philippe Boula de Mareuil, Catherine Brissaud, Aude Bretegnier, Maria Candea, Véronique Castellotti, Régine Delamotte, Jean-François De Pietro, Catherine Delarue-Breton, Anne Dister, Alexandre Duchêne, Valentin Feussi, Françoise Gadet, Stéphanie Galligani, François Gaudin, Monica Heller, Emmanuelle Huver, Caroline Juilliard, Abou Bakri Kebe, Jean-Marie Klinkenberg, Christian Lagarde, Foued Laroussi, Fabienne Leconte, Evelyne Lloze, Nolwenn Lorenzi, Gudrun Ledegen, Nadja Maillard, Marinette Matthey, Bruno Maurer, Véronique Miguel Addisu, Saskia Mugnier, Claire Oger, Marielle Rispail, Richard Sabria, Véronique Traverso, Christel Troncy, Cécile Van den Avenne. 